\title{
LEXICAL CORRESPONDENCES BETWEEN PROTO-KUKI-CHIN, HAKHA LAI, AND SIX MARAIC VARIETIES
}

June, 2020

Samson Lotven

Indiana University Department of Linguistics

\begin{abstract}
The list presented here is intended as an elicitation program for future research on Maraic varieties of Kuki-Chin. As such, it includes both data used to create the elicitation instrument and data collected using it. The priority in this list is to maximize the possibility of eliciting different rhyme types for comparative analysis purposes.
\end{abstract}

Key Terms - Kuki-Chin, Zophei, Lutuv, Mara, Zotung, Hakha Lai, Lexical correspondences

\section{LIST DESCRIPTION}

In order to facilitate future research on Kuki-Chin languages, especially on Maraic varieties, this paper offers lexical correspondences between several synchronic Kuki-Chin languages organized by Proto-Kuki-Chin etyma to facilitate lexical comparison. The sources of data included in that list are varied. Proto-Kuki-Chin reconstructions, Hakha Lai, and Mara items are all from VanBik (2009); Tlawngrang, Lawngtlang, and Nuitah Zophei, as well as Hnaring Lutuv items are all from field data collected by the author, and Zotung data is from Shintani, 2016. Apart from Hakha Lai, all varieties included here are considered Maraic in VanBik (2009)

The original elicitation instrument was created by comparing Tlawngrang and Lawngtlang Zophei field data from Appendix A with VanBik (2009). There were several principles used in creating the list:

1) Include at least one of each Proto-Kuki-Chin rhyme type. This principle prioritizes identifying diachronic rhyme change through comparison of Proto-Kuki-Chin rhymes with any other data sets added to the list.

2) For each Proto-Kuki-Chin rhyme type, give priority to lexical items found in both varieties of Zophei. This principle prioritizes lexical items likely to be more widely known between Zophei varieties. 
3) For each Proto-Kuki-Chin rhyme type, include all different Zophei rhymes. This principle prioritizes inclusion of lexical items where further elicitation will likely encounter variation.

4) For lexical items found in both varieties of Zophei, give priority to those with a correspondence in Mara. Mara is the most closely related language to Zophei in VanBik (2009), so prioritizing correspondences with those data facilitates interaction with synchronic data. It also promotes inclusion of lexical items likely to be found in Mara and other Marainfluenced varieties.

To the Proto-Kuki-Chin reconstruction, the Tlawngrang and Lawngtlang Zophei data sets, and the Mara data from VanBik (2009), four additional data sets were added: Hakha Lai data from VanBik (2009), Zotung data from Shintani (2016), and field data from Hnaring Lutuv and Nuitah Zophei that was collected using this instrument with the help of Sui Hnem Par and Kimberly Sakhong, respectively. Following are descriptions of those data sets and their inclusion in the data set.

Proto-Kuki-Chin reconstruction. This list builds off of VanBik's (2009) reconstructions with an eye towards future elicitation using the list. Using the widest variety of reconstructed rhymes offers future field workers the best chances of quickly finding different paths of diachronic rhyme development and determining what rhymes are in a language. Reconstructions are marked with an asterisk $(*)$.

Lawngtlang Zophei. These data were elicited using VanBik (2009) as part of a larger project from Spring 2018 to Summer 2020 with the help of Zai Sung, a 23-year-old native speaker from Lawngtlang. She currently lives in Indianapolis, IN. Note that in Lawngtlang Zophei final glottal stop is marked with $<\mathrm{h}>$.

Tlawngrang Zophei. These data were elicited using VanBik (2009) as part of a larger project from Spring 2018 to Spring 2019 with the help of Thomas Thawngza, a 22-year-old native speaker born outside of Tlawngrang to Zophei-speaking parents from Tlawngrang and Zephai (reportedly the same variety of Zophei). His family moved back to Tlawngrang when he was young. He currently lives in Indianapolis, IN. Note that in Tlawngrang Zophei, final glottal stop is marked with $<\mathrm{h}>$.

Mara. The data used for this data set are from VanBik (2009), the only Maraic language included there. In order to facilitate direct comparison with the text, Mara data were prioritized. Though VanBik (2009) relies on Mara orthography, he also notes where pronunciations do not mirror orthographic conventions. For those lexical items, I have included the phonetic transcription rather than the orthography.

Hakha Lai. Much of VanBik's (2009) Proto-Kuki-Chin rhyme inventory can be found in Hakha Lai, so inclusion of Hakha Lai allows for synchronic comparison. Also, many Maraic language speakers also speak Hakha Lai, making it a useful stimulus list in elicitation. The orthography used is from the source text.

Zotung. Shintani's (2016) word list is the only available information on Zotung and its inclusion offers the first diachronic comparison between Zotung and other Kuki-Chin languages. The orthography is modified from the source text to indicate tone and nasality as diacritics where Shintani (2016) uses numbers to mark tone and final $<\mathrm{n}>$ to mark nasality. 
Nuitah Zophei. These data were elicited in Spring 2020 using this instrument with the help of Kimberly Sakhong, a 21-year-old native speaker from Nuitah (Leitak). Kimberly was born to Zophei-Speaking parents in Nuitah, then moved to Hakha when she was a baby, to Malaysia when she was 8, and to Indianapolis (where she currently resides) when she was 10 .

Hnaring Lutuv. These data were elicited using this instrument from Spring 2019 to Spring 2020 as part of a larger project with the help from Sui Hnem Par, a 22-year-old native speaker born to Lutuv-speaking parents outside of Hnaring. Her family moved back to Hnaring when she was 7. She currently lives in Indianapolis, IN. Tone is not included in Lutuv transcriptions since little is yet known about the Lutuv tone system.

Special thanks goes out to all of the language assistants consulted in the creation of this list. Their patience and commitment made this research possible. Any errors in transcription are my own.

\section{LEXICAL CORREPONDENCE TABLE}

\begin{tabular}{|c|c|c|c|c|c|c|c|c|c|}
\hline & PKC & H. Lai & TRZ & NTZ & LTZ & $\begin{array}{l}\text { Hnaring } \\
\text { Lutuv }\end{array}$ & Mara & Zotung & Gloss \\
\hline 1. & *khlii & thlîi & thlìì & thlìt & thlì̀ & thlii & thlì & $\begin{array}{l}\text { khì væ̀ } \\
\text { khì sã̃ }\end{array}$ & wind \\
\hline 2. & ${ }^{*}$ kri? & tri? & tsíí & tsít & tsín & tsï & chí & rí & to fear \\
\hline 3. & *hniit & $\begin{array}{l}\text { hniit-I } \\
\text { hni?-II }\end{array}$ & hníí & hnít & hnútí & hning & hnó & mõ & $\begin{array}{l}\text { to blow } \\
\text { (one's nose) }\end{array}$ \\
\hline 4. & $\begin{array}{l}{ }^{*} \text { phiat } \\
\text { or *phiak }\end{array}$ & $\begin{array}{l}\text { phiak-I } \\
\text { phia?- } \\
\text { II }\end{array}$ & phíí & phíá & phít & $\begin{array}{l}\text { phï } \\
{\left[p^{\mathrm{h}} \mathrm{sii}\right]}\end{array}$ & phí & phí & to sweep \\
\hline 5. & $*_{\text {kil }}$ & kìl & kíí & kín & kíf & $\mathrm{kii}$ & kí & sà kí & $\begin{array}{l}\text { angle, } \\
\text { corner, horn }\end{array}$ \\
\hline 6. & *ruul & rûul & pá rìì & pá rì̀ & pá rì̀ & pa rii & pā-rì & hù pò & snake \\
\hline 7. & *kul & kûl & sáng kì̀ & sáng kì̀ & $\begin{array}{l}\text { sáng } \\
\text { kì̀ }\end{array}$ & soo kii & pa-kì & $\theta$ à̀ kứ & twenty \\
\hline 8. & *bia & bǐa & bíá & bì̀ & bíé & bie & $\mathrm{b} \overline{1}$ & búuá & word \\
\hline 9. & $*$ hrial & $\begin{array}{l}\text { hrîal-I } \\
\text { hrial-II }\end{array}$ & tsá hrì̀ & hrìà & $\begin{array}{l}\text { tsá } \\
\text { hrìè }\end{array}$ & tsa hrï & $\begin{array}{l}\text { chā- } \\
\text { hrì }\end{array}$ & - & to avoid \\
\hline 10. & *pher & phêr & á phìà & a phìà & phì̀ & a phaa & - & phìà & mat \\
\hline 11. & *mer? & mer? & mìà & mì̀̀ & míé & tsa maa & $\begin{array}{l}\text { chā- } \\
\text { mì }\end{array}$ & - & to twist \\
\hline 12. & $\begin{array}{l}\text { *ha? } \\
\text { thiaw }\end{array}$ & $\begin{array}{l}\text { ha?- } \\
\text { thǐaw }\end{array}$ & há thíó & há thì̀ & $\begin{array}{l}\text { há } \\
\text { thíú }\end{array}$ & ha thum & há-thī & hà chí & to sneeze \\
\hline 13. & *hrik & hrik & hrìh & hrìh & hríh & a hrï & hrī & 6í? & louse \\
\hline 14. & $\begin{array}{l}*_{\text {kut }} \\
\text { (or } \\
\text { *khut) }^{\text {kn }}\end{array}$ & kut & kìh & kìh & kìh & kut & $\mathrm{kū}$ & kúí? & hand \\
\hline 15. & *?im & Pîn & íng & íng & íng & ing & ó & Pर́ & house \\
\hline
\end{tabular}


Indiana Working Papers in South Asian Languages and Cultures

\begin{tabular}{|c|c|c|c|c|c|c|c|c|c|}
\hline 16. & $*_{\text {riin }}$ & rüin & ríng & ríng & ríng & tsa rii & rò & - & $\begin{array}{l}\text { to make a } \\
\text { line }\end{array}$ \\
\hline 17. & $*_{\text {thin }}$ & thǐn & thíng & thíng & thíng & thii & pa-thī & phĩ̀ & liver \\
\hline 18. & $*$ thiin & thîin & èè síng & àì sìng & $\begin{array}{l}\text { è̀̀ } \\
\text { sìng }\end{array}$ & ie sing & - & - & ginger \\
\hline 19. & *thin & thîn & thíng & thíng & thíng & thing & thó & thî́ & tree, wood \\
\hline 20. & $*_{\text {kir }}$ & $\begin{array}{l}\text { kîr-I } \\
\text { kǐr-II }\end{array}$ & kíng & kìng & kìng & kəng & $\begin{array}{l}\text { chā- } \\
\text { kú }\end{array}$ & kòè & to curl \\
\hline 21. & *hley & $\begin{array}{l}\text { hlêy-I } \\
\text { hlěn-II }\end{array}$ & hìng & hìng & hìng & həng & - & $\begin{array}{l}\text { lî́ 'cheat' } \\
\text { 'che hǜ } \\
\text { 'lie' }\end{array}$ & to lie, cheat \\
\hline 22. & $*$ doon & - & díng & díng & díng & dəng & dò & - & to drink \\
\hline 23. & *yuum & $\begin{array}{l}\text { zŭum-I } \\
\text { zum?- } \\
\text { II }\end{array}$ & zíng & zíng & zíng & zəng & zó & 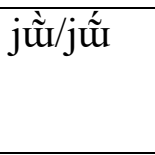 & to believe \\
\hline 24. & *tum & $\begin{array}{l}\text { tûm-I } \\
\text { tǔm-II }\end{array}$ & tíng & tíng & tìng & - & - & - & to intend \\
\hline 25. & *phun & phǔn & phíng & phíng & phíng & phəng & phō & - & clan, kind \\
\hline 26. & $\begin{array}{l}\text { *hnuy } \\
\text { (or *nuy) }\end{array}$ & $\begin{array}{l}\text { hma- } \\
\text { nùn }\end{array}$ & hnìng & hnì̀ & hníng & hnəng & - & nù lấ & back \\
\hline 27. & $*_{\text {nii }}$ & nîi & nìng & nì & nín & ning & nò & $\begin{array}{l}\text { nĩ́ (sun) } \\
\text { nĩ } \\
\text { (day) }\end{array}$ & day, sun \\
\hline 28. & $\begin{array}{l}*_{\mathrm{hmul}} \\
\text { (or *mul) }\end{array}$ & hmûl & hmíng & hmíń & hmín & hming & - & mî́ & hair \\
\hline 29. & *rian & rǐan & hréé & hràì & hréé & hrie & rāi & rúuá & to work \\
\hline 30. & $*$ lee & lěe & léé & léé & léé & hnee & - & té & and, with \\
\hline 31. & ${ }^{*}$ we? & ve? & véé & váí & véé & vee & - & pæ̀ bùà & to visit \\
\hline 32. & $*$ hmeel & - & hméé & hmáí & hméé & hmie & - & mǽ & face \\
\hline 33. & *yal & yâl & $\begin{array}{l}\text { ngèè } \\
\text { láng }\end{array}$ & ngèè & $\begin{array}{l}\text { ngéé } \\
\text { láng }\end{array}$ & ngie luo & $\begin{array}{l}\text { ngìà } \\
\text { lý }\end{array}$ & - & shin \\
\hline 34. & *waay & $\begin{array}{l}\text { văay-I } \\
\text { vay?-II }\end{array}$ & vèè & - & vèè & vie & $\begin{array}{l}\text { pā- } \\
\text { vīa }\end{array}$ & - & to stray \\
\hline 35. & $*$ tay & $\begin{array}{l}\text { tây-I } \\
\text { tǎy-II }\end{array}$ & téé & táí & téé & tee & tèi & tæ & to win \\
\hline 36. & *phooy & $\begin{array}{l}\text { phôoy- } \\
\text { I } \\
\text { phǒoy- } \\
\text { II }\end{array}$ & phéé & pháí & phéé & phie & phía & - & to uproot \\
\hline 37. & *pa-sal & pa-sâl & pa séé & pa séé & pá séí & - & - & - & husband \\
\hline 38. & *khaaw & $\begin{array}{l}\text { khăaw- } \\
\text { bok }\end{array}$ & $\begin{array}{l}\text { khéé } \\
\text { bàh }\end{array}$ & - & $\begin{array}{l}\text { khǿǿ } \\
\text { bìh }\end{array}$ & $\begin{array}{l}\text { khoo } \\
\text { bшә рәә }\end{array}$ & - & - & grasshopper \\
\hline 39. & *raal & râal & rèì & rèè & réí & tsa rie & $\begin{array}{l}\text { chā- } \\
\text { rìa }\end{array}$ & rò & enemy, war \\
\hline 40. & *?al & Pâl-I & èì & èè & èì & ie & ìa & Pa Pè & to be salty \\
\hline
\end{tabular}


Indiana Working Papers in South Asian Languages and Cultures

\begin{tabular}{|c|c|c|c|c|c|c|c|c|c|}
\hline & & Păl-II & & & & & & & \\
\hline 41. & $*$ tel & $\begin{array}{l}\text { tèl-I } \\
\text { těl-II }\end{array}$ & tèì & tèè & tèì & tee & - & - & to participate \\
\hline 42. & *phay & $\begin{array}{l}\text { phây-I } \\
\text { phăy-II }\end{array}$ & phè̀̀ & phàì & phèè & phie & - & - & to be flat \\
\hline 43. & $*_{\text {mee }}$ & me-he? & méí & mií & míí & mie & $\mathrm{mi}$ & níá & goat \\
\hline 44. & $* \theta \mathrm{e} ?$ & - & sèì & síí & síi & sii & Sî̀ & $\theta$ é? & to go \\
\hline 45. & *6eel & bêel & bèì & bì̀ & bíí & bii & bèi & - & pot \\
\hline 46. & *mool & $\begin{array}{l}\text { mòol- } \\
\text { mok-I } \\
\text { móol- } \\
\text { mok-II }\end{array}$ & mèì & mì̀ & mì̀ & mயә & - & Pa yúé? & to be dull \\
\hline 47. & *riat & pa-riat & $\begin{array}{l}\text { sáng } \\
\text { réh }\end{array}$ & són rèh & $\begin{array}{l}\text { sàng } \\
\text { réh }\end{array}$ & $\begin{array}{l}\text { suo tsa } \\
\text { rii }\end{array}$ & $\begin{array}{l}\text { pā- } \\
\text { chā-rí }\end{array}$ & tá rùà̀ & eight \\
\hline 48. & $*_{\text {mik }}$ & mit & mèh & mih & mìh & ming & $\mathrm{mō}$ & mí? & eye \\
\hline 49. & $\begin{array}{l}\text { *tiap } \\
\text { (or } \\
\text { *tiam) }\end{array}$ & $\begin{array}{l}\text { tep-I } \\
\text { te?-II }\end{array}$ & pá tèh & tèh & pá tìh & - & - & Pæ̇ tùà & to taste \\
\hline 50. & *leet & leet & lèh & lèh & pá lìh & - & - & líá & $\begin{array}{l}\text { to change } \\
\text { (one's mind) }\end{array}$ \\
\hline 51. & *?eek & Peek & éh & èh & ih & ii & - & Píá & feces \\
\hline 52. & $\begin{array}{l}\text { *sat-II } \\
\left(*_{\text {saa-I }}\right)\end{array}$ & $\begin{array}{l}\text { sâa-I } \\
\text { sat-II }\end{array}$ & sèh & sèh & sìh & Səว & sà & Pa sò & to be hot \\
\hline 53. & $*_{\text {tew? }}$ & tew? & tǿǿ & tóí & tǿǿ & pa-tii & pa-tei & thæ & to bite \\
\hline 54. & *kaar & kăar & tsá kø̀ø & kàà & $\begin{array}{l}\text { tsá } \\
\text { kø̀ø̀ }\end{array}$ & - & - & - & to separate \\
\hline 55. & *ray & - & rǿǿ & róí & rǿǿ & ruu & rēi & Pa ròù & $\begin{array}{l}\text { to take a } \\
\text { long time }\end{array}$ \\
\hline 56. & *maaw & mâaw & rá mø̀ø & - & $\begin{array}{l}\text { rà } \\
\text { mǿǿ }\end{array}$ & aa rï & rā-mò & - & bamboo \\
\hline 57. & *baw & bàw & bø̀ø & bòì & bø̀ø & buu & bàù & - & $\begin{array}{l}\text { to swell, to } \\
\text { bump up }\end{array}$ \\
\hline 58. & *khoy? & khoy? & khǿǿ & khùù & khǿǿ & khyə & - & - & to dig, touch \\
\hline 59. & $\begin{array}{l}* \text { yuan-I } \\
* \text { yuan-II }\end{array}$ & $\begin{array}{l}\text { zûay-I } \\
\text { zûan-II }\end{array}$ & zø̀ø & zóí & zø̀ø & zuu & á zó & Pa jù & to fly \\
\hline 60. & *lay-baa & lây-bàa & lúí bàà & lùì bòò & $\begin{array}{l}\text { lỳỳ } \\
\text { báá }\end{array}$ & lyy buu & lì-bà & læ̀ & debt \\
\hline 61. & *tsaar & $\begin{array}{l}\text { tsâar-I } \\
\text { tsăar-II }\end{array}$ & tsàà & tsàà & tsàà & tsaa & chá & - & to be dry \\
\hline 62. & *thar & $\begin{array}{l}\text { thâr-I } \\
\text { thăr-II }\end{array}$ & thàà & thàà & thàà & thaa & thì & ?a thò & to be new \\
\hline 63. & $\begin{array}{l}\left.{ }^{*} \text { woor-I }\right) \\
\left({ }^{*} \text { wor?- }\right. \\
\text { II })\end{array}$ & $\begin{array}{l}\text { vòr?-I } \\
\text { vôr?-II }\end{array}$ & váá & váá & váá & vaa & - & vùà & to throw (at) \\
\hline 64. & *thiam & $\begin{array}{l}\text { thîam-I } \\
\text { thǐam- }\end{array}$ & tháí & théng & théng & thee & thái & $\begin{array}{l}\text { thǽ } \\
\text { thúuá }\end{array}$ & $\begin{array}{l}\text { to be skillful } \\
\text { in }\end{array}$ \\
\hline
\end{tabular}


Indiana Working Papers in South Asian Languages and Cultures

\begin{tabular}{|c|c|c|c|c|c|c|c|c|c|}
\hline & & II & & & & & & & \\
\hline 65. & *rian & rǐan & ráí & réng & réng & ree & rāi & rúuá ló & work \\
\hline 66. & *liay & lîan & làì & lèng & lèng & lee tung & - & - & shoulder \\
\hline 67. & *peem & $\begin{array}{l}\text { pêem-I } \\
\text { pěem- } \\
\text { II }\end{array}$ & pàì & pèng & pèng & pee & pài & píá & to migrate \\
\hline 68. & *hrem & $\begin{array}{l}\text { hrêm-I } \\
\text { hrěm- } \\
\text { II }\end{array}$ & hráí & hréng & hréng & hrii & hrí & - & $\begin{array}{l}\text { to torture } \\
\text { to punish }\end{array}$ \\
\hline 69. & $* \mathrm{kh}(\mathrm{r}) \mathrm{en}$ & $\begin{array}{l}\text { khên-I } \\
\text { khěn-II }\end{array}$ & tsháí & tshéng & tshèng & tshee & chhài & $\begin{array}{l}\text { múá } \\
\text { kúú }\end{array}$ & $\begin{array}{l}\text { to separate, } \\
\text { to get } \\
\text { divorced }\end{array}$ \\
\hline 70. & $*$ tleen & tlen? & tláí & tléng & tléng & pa tlee & - & $\begin{array}{l}\text { Pa } \\
\text { thùuà }\end{array}$ & $\begin{array}{l}\text { to pour in, } \\
\text { rinse }\end{array}$ \\
\hline 71. & *bey & $\begin{array}{l}\text { bèn-I } \\
\text { běn-II }\end{array}$ & báí & béng & béng & - & - & - & to compress \\
\hline 72. & $*$ tsam & tsăm & tsáí & tséng & tséng & dzuo & - & - & occasion \\
\hline 73. & *haan & $\begin{array}{l}\text { hâan-I } \\
\text { hăan-II }\end{array}$ & hàì & héng & hèng & - & hía & húé væ̀ & to cease \\
\hline 74. & $* \tan$ & $\begin{array}{l}\text { tân-I } \\
\text { tăn-II }\end{array}$ & táí & téng & téng & tee & tái & - & to cut off \\
\hline 75. & *daw & $\begin{array}{l}\text { dâw-I } \\
\text { dăw-II }\end{array}$ & dáí & déng & déng & tuu dee & dàù & dúu kú & to fight \\
\hline 76. & *roon & $\begin{array}{l}\text { rǒon-I } \\
\text { ron?-II }\end{array}$ & ráí & réng & réng & roo & rỳ & - & to pour in \\
\hline 77. & $\begin{array}{l}\text { *hlaay } \\
\text { (or *lay) }\end{array}$ & $\begin{array}{l}\text { hlâay-I } \\
\text { hlǎay- } \\
\text { II }\end{array}$ & hláí & hláí & hléé & - & - & - & to operate \\
\hline 78. & *thaw & $\begin{array}{l}\text { thâw-I } \\
\text { tho?-II }\end{array}$ & thàù & thúú & thùù & thuu & thú & $\begin{array}{l}\text { thǽ kú } \\
\text { thó kú }\end{array}$ & to get up \\
\hline 79. & *?oo & Pòo & áú & úú & úú & уә & ú & - & voice \\
\hline 80. & $*_{\text {so? }}$ & so? & sáú & súú & súú & - & sù & - & to pierce \\
\hline 81. & *hroop & $\begin{array}{l}\text { hroop-I } \\
\text { hro?-II }\end{array}$ & hràù & hrúú & hrùù & pa hrii & - & - & $\begin{array}{l}\text { to eat } \\
\text { (porridge) }\end{array}$ \\
\hline 82. & *hrol? & hrol? & hráú & hrúú & hrúú & - & - & - & to poke \\
\hline 83. & $*_{\text {zoy }}$ & $\begin{array}{l}\text { zôy-I } \\
\text { zǒy-I }\end{array}$ & zàù & zùù & zùù & zуә & - & - & to be skinny \\
\hline 84. & $*$ nuu & nûu & náú & nùù & núú & nung & nó & nố & mother \\
\hline 85. & $\begin{array}{l}* \text { hruk } \\
\text { (or *ruk) }\end{array}$ & hruk & hràù & hrúú & hrùù & hruu & - & - & $\begin{array}{l}\text { to put on } \\
\text { clothes }\end{array}$ \\
\hline 86. & *yum & $\begin{array}{l}\text { zûm-I } \\
\text { zŭm-II }\end{array}$ & pá zàù & zùng & $\begin{array}{l}\text { pá } \\
\text { zùù }\end{array}$ & - & pa-zo & Pa jữ & $\begin{array}{l}\text { to be sharp, } \\
\text { pointed }\end{array}$ \\
\hline 87. & *hmuur & hmǔur & hmáú & hmúú & hmúú & hmuu & hmū & mõ̃ & mouth \\
\hline 88. & *tuuy & - & thàù & thùù & thùù & - & - & $\begin{array}{l}\text { múuá } \\
\text { rùùa } \\
\text { thùà }\end{array}$ & to be tasty \\
\hline
\end{tabular}


Indiana Working Papers in South Asian Languages and Cultures

\begin{tabular}{|c|c|c|c|c|c|c|c|c|c|}
\hline 89. & $\begin{array}{l}* \text { dua-I } \\
(* \text { dua-II })\end{array}$ & $\begin{array}{l}\text { dǒo-I } \\
\text { doot-II }\end{array}$ & dáú & dúú & dúú & dyə & - & - & to love \\
\hline 90. & $\begin{array}{l}\text { *phrat-II } \\
(* \text { phraa- } \\
\text { I) }\end{array}$ & $\begin{array}{l}\text { thră-I } \\
\text { thrat-II }\end{array}$ & tshàh & tshàh & tshàh & phəə & phā & - & to be good \\
\hline 91. & *khaak & khaak & khàh & khàh & kháh & $\begin{array}{l}\text { duo } \\
\text { khaa }\end{array}$ & $\begin{array}{l}\text { dà- } \\
\text { khó }\end{array}$ & - & phlegme \\
\hline 92. & $\begin{array}{l}*_{\text {*sak-II }} \\
\left({ }^{*} \text { saa-I }\right)\end{array}$ & $\begin{array}{l}\text { săa-I } \\
\text { sak-II }\end{array}$ & sàh & sàh & sáh & səə & sā & sà? & to build \\
\hline 93. & $\begin{array}{l}* \text { lem-I } \\
(* \text { lem?- } \\
\text { II) }\end{array}$ & lem? & láng & láng & láng & - & - & - & to swallow \\
\hline 94. & *laam & $\begin{array}{l}\text { lâam-I } \\
\text { lăam-II }\end{array}$ & làng & làng & làng & luo & là & Pa lò & to dance \\
\hline 95. & $*^{2} \mathrm{~s}^{\mathrm{h}} \mathrm{am}$ & sâm & sáng & sáng & sáng & suo & sá & sã́ & hair (head) \\
\hline 96. & *baan & bâan & bàng & bèng & báng & buo & bá & - & arm \\
\hline 97. & $\begin{array}{l}\text { *klan-I } \\
(* \text { klan?- } \\
\text { II) }\end{array}$ & $\begin{array}{l}\text { tlăn-I } \\
\text { tlan?-II }\end{array}$ & tláng & tláng & tláng & aa thləə & $\begin{array}{l}\text { chā- } \\
\text { tlāi }\end{array}$ & - & to redeem \\
\hline 98. & *klaay & tlâay & tlàng & tlàng & tlàng & tluo & tlà & - & $\begin{array}{l}\text { hill, } \\
\text { mountain }\end{array}$ \\
\hline 99. & *dan & dân & dàng & dàng & dàng & duo & dà & - & palate \\
\hline 100. & $*_{\text {thiir }}$ & $\begin{array}{l}\text { thîir } \\
\text { (or } \\
\text { thîar) }\end{array}$ & thóó & thóó & tháú & thaa & thúá & - & metal \\
\hline 101. & *thaa & $\begin{array}{l}\text { thâay-I } \\
\text { thăay- } \\
\text { II }\end{array}$ & thóó & thóó & tháú & thaa & thá & thò jố & strength \\
\hline 102. & $*$ ta? & ta? & tóó & tóó & táú & taa & - & tó & to measure \\
\hline 103. & *hnaar & hnăar & hnóó & hnóó & hnáú & $\begin{array}{l}\text { hnaa tsa } \\
\text { but }\end{array}$ & - & nà & nose, sinus \\
\hline 104. & *yon & $\begin{array}{l}\text { yòy-I } \\
\text { yŏn-II }\end{array}$ & ngòò & ngóó & ngàù & ngaa & - & - & to be stupid \\
\hline 105. & *klor & $\begin{array}{l}\text { tlôr-I } \\
\text { tlǒr-II }\end{array}$ & tlóó & tlòì & tláú & tlyə & tlù & - & to be oily \\
\hline 106. & *rook & $\begin{array}{l}\text { rook-I } \\
\text { ro?-II }\end{array}$ & ròh & ràh & ráh & rəə & rú & - & $\begin{array}{l}\text { to break } \\
\text { down }\end{array}$ \\
\hline 107. & ${ }^{*}$ wok & vok & vòh & vàh & váh & vəว & $\mathrm{vu \overline {u }}$ & vóú? & pig \\
\hline 108. & $*$ tsuap & tsuap & tsòh & tsùh & tsùh & pa tsəə & $\begin{array}{l}\text { pā- } \\
\text { chú }\end{array}$ & túá & lung \\
\hline 109. & *hnim & $\begin{array}{l}\text { hnîm-I } \\
\text { hnǐm- } \\
\text { II }\end{array}$ & nóng & nóng & nóng & noo & - & - & to dip \\
\hline 110. & *6oom & bôom & bòng & bòng & bóng & boo & bỳ & hé? bõ̀ & box, basket \\
\hline 111. & *pom & $\begin{array}{l}\text { pôm-I } \\
\text { pǒm-II }\end{array}$ & póng & pòng & póng & poo & pù & põ̀ & to embrace \\
\hline
\end{tabular}


Indiana Working Papers in South Asian Languages and Cultures

\begin{tabular}{|c|c|c|c|c|c|c|c|c|c|}
\hline 112. & *hoon & hôon & hòng & hòng & hóng & hoo & hỳ & Pa fú & cover \\
\hline 113. & $\begin{array}{l}* \text { doy } \\
\text { (or *toy) }\end{array}$ & dǒn & dòng & dóng & dóng & - & dó & - & cubit \\
\hline 114. & *kol? & kol? & kòng & kóng & kòng & - & - & - & to be bald \\
\hline 115. & $\begin{array}{l}\text { *nuam-I } \\
\left({ }^{*} \text { nuam?- }\right. \\
\text { II) }\end{array}$ & $\begin{array}{l}\text { nǔam-I } \\
\text { nuam?- } \\
\text { II }\end{array}$ & nòng & nóng & nòng & nuo & - & nò $\theta$ ó & $\begin{array}{l}\text { to be happy, } \\
\text { comfortable }\end{array}$ \\
\hline 116. & *khuay & khûay & khòng & khòng & khòng & aa khəy & khò & khúá & drum \\
\hline 117. & *yuu & zùu & pá zúú & pà zúú & $\begin{array}{l}\text { pá } \\
\text { zúúu }\end{array}$ & pa zut & $\begin{array}{l}\text { pā- } \\
\text { zúu }\end{array}$ & pà jú & rat, mouse \\
\hline 118. & *du? & du? & dúú & dúú & dúú & - & $\mathrm{du \overline {u }}$ & - & to want \\
\hline 119. & $\begin{array}{l}\text { *suum } \\
\text { (or } \\
\text { *siim) }^{\text {siim }}\end{array}$ & sǔum & pá súú & pà súú & pà súú & pa tuu & - & tùà & to grasp \\
\hline 120. & *kuul & $\begin{array}{l}\text { kûul-I } \\
\text { kǔul-II }\end{array}$ & kùù & kùù & kùù & kung & - & - & $\begin{array}{l}\text { to be } \\
\text { hunchbacked }\end{array}$ \\
\hline 121. & $\begin{array}{l}\text { *hmu?-II } \\
(* \mathrm{hmuu}- \\
\mathrm{I})\end{array}$ & $\begin{array}{l}\text { hmǔu-I } \\
\text { hmu?- } \\
\text { II }\end{array}$ & hmùh & hmùh & hmúh & hmung & hmō & múí? & to see \\
\hline 122. & *?uut & $\begin{array}{l}\text { Puut-I } \\
\text { Pu?-II }\end{array}$ & ùh & ùh & ùh & $\mathrm{HU}$ & úu & Pù & to burn \\
\hline 123. & *thuuk & $\begin{array}{l}\text { thuuk-I } \\
\text { thu?-II }\end{array}$ & thùh & thùh & thùh & thet & thú & - & to be deep \\
\hline 124. & *ruk & pa-ruk & $\begin{array}{l}\text { sáng } \\
\text { rúh }\end{array}$ & sán rùh & $\begin{array}{l}\text { sàng } \\
\text { rúh }\end{array}$ & $\begin{array}{l}\text { suo tsa } \\
\text { ruw }\end{array}$ & $\begin{array}{l}\text { pā- } \\
\text { chá- } \\
\text { rū }\end{array}$ & tá rú? & six \\
\hline 125. & $\begin{array}{l}*_{\text {puak-II }} \\
(* \text { pua-I })\end{array}$ & $\begin{array}{l}\text { pùa-I } \\
\text { puak-II }\end{array}$ & pùh & pùh & púh & puru & $\mathrm{p} \overline{\mathbf{1}}$ & - & $\begin{array}{l}\text { to carry a } \\
\text { person (on } \\
\text { back) }\end{array}$ \\
\hline 126. & *kup & $\begin{array}{l}\text { kup-I } \\
\text { ku?-II } \\
\end{array}$ & tsá kúú & kúú & $\begin{array}{l}\text { tsá } \\
\text { kúh }\end{array}$ & - & - & - & $\begin{array}{l}\text { to surround, } \\
\text { to embrace }\end{array}$ \\
\hline 127. & *lay & lây & lùì & lùì & lýý & pa lyy & pā-lei & læ̀ & tongue \\
\hline 128. & *hool & $\begin{array}{l}\text { hôol-I } \\
\text { hǒol-II }\end{array}$ & húí & húí & hýý & hut & - & - & to search for \\
\hline 129. & *6ooy & bòoy & búí & búí & býý & byy & béi & - & master \\
\hline 130. & *poy & $\begin{array}{l}\text { pòy- } \\
\text { mo?-I } \\
\text { pǒy- } \\
\text { mo?-II }\end{array}$ & $\begin{array}{l}\text { púí } \\
\text { mòò }\end{array}$ & púí & $\begin{array}{l}\text { pýý } \\
\text { màù }\end{array}$ & руу рәә & - & - & to matter \\
\hline 131. & *ruuy & $\begin{array}{l}\text { rǐi-I } \\
\text { riit-II }\end{array}$ & rúí & rúí & rýý & pa rï & pā-rī & rùì & to be drunk \\
\hline 132. & $*$ tuy & tîi & túí & tuí & týý & tiii & tí & túí & water \\
\hline 133. & *?uy? & Puy? & ùì & uí & ỳỳ & - & - & - & to be rotten \\
\hline 134. & *tuay & tûay & rá tùì & rà tùì & rá týý & a rii tyy & tè̀̀ & rù? túé & $\begin{array}{l}\text { edible } \\
\text { bamboo }\end{array}$ \\
\hline 135. & $*$ kul-I & kul? & tsá kúá & kúá & tsà & - & - & kùè? lú & to surround \\
\hline
\end{tabular}


Indiana Working Papers in South Asian Languages and Cultures

\begin{tabular}{|c|c|c|c|c|c|c|c|c|c|}
\hline & $\begin{array}{l}(* \text { kul?- } \\
\text { II) }\end{array}$ & & & & kùà & & & & \\
\hline 136. & *khua & khûa & khùà & khùà & khúó & khum & khì & khùà & village \\
\hline 137. & $*$ tua? & tua? & túá & tùà & túó & tyə & tú & tùà & to do \\
\hline 138. & *tuak & tǔak & túá táng & túá táng & $\begin{array}{l}\text { thúó } \\
\text { táng }\end{array}$ & - & - & - & to count \\
\hline 139. & *hrual & $\begin{array}{l}\text { hrûal-I } \\
\text { hrǔal- } \\
\text { II }\end{array}$ & rúá & rúá & rùò & - & - & - & to roll \\
\hline 140. & *yuar & $\begin{array}{l}\text { zûar-I } \\
\text { zǔar-II }\end{array}$ & zúá & zúá & zùò & zaa & zùa & jùà & to sell \\
\hline 141. & $\begin{array}{l}\text { *puum } \\
\text { (or } \\
\text { *buum) }\end{array}$ & $\begin{array}{l}\text { pǔum-I } \\
\text { pum?- } \\
\text { II }\end{array}$ & pùng & púng & pùng & pəng & pō & Pa pû́ & $\begin{array}{l}\text { to be round, } \\
\text { to be piled }\end{array}$ \\
\hline 142. & *tum & tǔm & tùng & tùng & tùng & tong & tō & tỗ & block, post \\
\hline 143. & *yuun & yûun & ngúng & ngúng & ngúng & nguu & ngò & - & silver \\
\hline 144. & *yun & $\begin{array}{l}\text { zûy-I } \\
\text { zǔn-II }\end{array}$ & zúng & zúng & zúng & zung & pā-zō & Pa jữ̃̀̃ & to urinate \\
\hline 145. & *phuy & phûy & phùng & phùng & phùng & phung & phau & phõ̀ thó & custom \\
\hline
\end{tabular}

\section{REFERENCES}

Shintani, T. L. A. (2016). The Zotung Language. Tokyo: Research Institute for Languages and Cultures of Asia and Africa: Tokyo.

VanBik, K. (2009). Proto-Kuki-Chin: A Reconstructed Ancestor of the Kuki-Chin Languages. STEDT Monograph Series, Vol. 8.: University of California, Berkeley. 